VOL. 69 (2004) [395-401]

\title{
INVERSE SEMIGROUPS ALL OF WHOSE PROPER HOMOMORPHIC IMAGES ARE GROUPS
}

\author{
RALPH P. TUCCI
}

\begin{abstract}
We characterise those inverse semigroups whose proper (non-isomorphic) homomorphic images are all groups. We also show that the bicyclic semigroup is the only such semigroup in certain cases.
\end{abstract}

\section{INTRODUCTION}

The bicyclic semigroup is defined as $\mathcal{C}=\langle p, q \mid p q=1\rangle$. It is well known [2, Corollary 3.2], that every proper (non-isomorphic) homomorphic image of the bicyclic semigroup $\mathcal{C}$ is a group. (In fact, every proper homomorphic image of $\mathcal{C}$ is a cyclic group; however, we shall not use the cyclic property.) We shall refer to inverse semigroups all of whose proper homomorphic images are groups as $h$-groups; to eliminate certain trivial cases, we shall require that an $h$-group $S$ does have homomorphic images other than itself and the one-element semigroup, that $S$ is not a group, and that $S$ has more than two elements. In this paper we characterise $h$-groups in general. We also show that the bicyclic semigroup is the only $h$-group in certain cases.

There seem to be few published results on $h$-groups. Apparently Tamura [14] was the first person to ask about the structure of $h$-groups in his review of [1]. Bernstein has shown that $h$-groups are simple, and that they contain a copy of $\mathcal{C}$; see $[1$, Theorem 1.1 , Theorem 1.2, Corollary 1.3]. Fortunatov [3, Corollary 3] has given examples of $h$-groups which are similar to the bicyclic semigroup. Reilly [12, Lemma 3.3] has characterised the full inverse subsemigroups of $T_{E}$ which are $h$-groups, where $E$ is a semilattice which is a dense tree without zero and $T_{E}$ is the semigroup of all order isomorphisms between principal order ideals of $E$. Such semigroups have a modular lattice of congruences. Goberstein [4, Corollary 4.15] has proved that if an inverse semigroup has no idempotentseparating congruences and certain order relations are trivial, then that semigroup is an h-group. In a different vein, Munn [8] has characterised inverse semigroups which have no congruences except for the identity congruence and the universal congruence; these semigroups are known as congruence-free semigroups. As we shall see, $h$-groups are a natural generalisation of congruence-free inverse semigroups.

Received 17th September, 2003

Copyright Clearance Centre, Inc. Serial-fee code: $00049727 / 04 \quad \$ A 2.00+0.00$. 
More generally, several authors have studied semigroups whose congruences all share some property. Bernstein [1], Putcha [11], and Tamura and Hamilton [15] have studied semigroups such that every homomorphic image which is contained in a group is itself a group. Jensen [5] and Schein [13] have worked on semigroups $S$ whose non-trivial homomorphic images are all isomorphic to $S$. Zhu [17] has looked at semigroups whose non-identity congruences are all Rees congruences. Kowol and Mitsch [6] have studied finite Clifford semigroups whose non-trivial homomorphic images all have a non-trivial centre.

A semigroup $S$ is regular if for every $s \in S$ there is $t \in S$ such that $s=s t s$. An element $t \in S$ is an inverse of $s$ if $s=s t s$ and $t=t s t$. A semigroup $S$ is inverse if $S$ is regular and every element of $S$ has a unique inverse. It is known [2, Theorem 1.17] that a regular semigroup is inverse if and only if its idempotents commute with each other, which implies that the product of two idempotents is an idempotent. If $s \in S$ then we denote the inverse of $s$ by $s^{-1}$. It is easy to see that the bicyclic semigroup is inverse. In what follows every semigroup will be inverse. The set of idempotents is denoted $E$, and the cardinality of this set is denoted $|E|$. Note that, if $|E|=1$, then $S$ is a group. An inverse semigroup admits a partial order as follows: if $s, t \in S$, then $s \leqslant t$ if $s=e t$ for some $e \in E$.

A semigroup $S$ is congruence-free if every congruence on $S$ is either the universal congruence or the identity congruence. A group congruence is a congruence $\rho$ such that $S / \rho$ is a group. An idempotent separating congruence is a congruence $\rho$ such that $\rho \cap(E \times E)$ is the identity congruence on $E$. Every inverse semigroup has a unique maximal idempotent-separating congruence $\mu$ and a unique minimal group congruence $\sigma$; see $[9$, p. 131, p. 142$]$.

If $\rho$ is a congruence, then the kernel of $\rho$ is the set $\operatorname{ker} \rho=\{s \in S \mid$ spe for some $e \in E\}$. The trace of $\rho$ is the restriction of $\rho$ to the set of idempotents, denoted tr $\rho$. The centraliser of the set of idempotents is the set $C(E)=\{s \in S \mid s e=e s$ for all $a \in E\}$.

\section{The General Case}

An inverse semigroup $S$ is an $h$-group if $S$ is not congruence-free, $S$ is not a group, $|S|>2$, and every proper homomorphic image of $S$ is a group.

Note that every semigroup with two elements has the one-element group as its only proper homomorphic image; for this reason, we eliminate this case from the definition. We need the next result to characterise $h$-groups.

THEOREM 1. If $S$ is an inverse semigroup then ker $\mu=C(E)$.

Proof: This is found in [7, Proposition 3, p. 139] or [9, Theorem III.3.5]. 
Theorem 2 is based on the characterisation of congruence-free inverse semigroups due to Munn in [8]. The proof of Theorem 2 follows that of [9, Theorem IV.3.3] almost exactly.

THEOREM 2. Let $S$ be an inverse semigroup such that $|E|>1$; that is, $S$ is not a group. Then $S$ is an $h$-group if and only if $S$ satisfies the following conditions:

(a) $C(E)=E$;

(b) for any $e, f, g, h \in E$ with $e>f$ and $g>h$, there exist $t_{1}, t_{2}, \ldots, t_{n} \in S$ such that $g=t_{1}^{-1} e t_{1}, t_{i}^{-1} f t_{i}=t_{i+1}^{-1} e t_{i+1}$ for $1 \leqslant i<n$, and $t_{n}^{-1} f t_{n} \leqslant h$.

Proof: Let $S$ be an $h$-group. By supposition there exist $e \neq f$ in $S$; hence $e \mu \neq f \mu$ and so $S / \mu$ cannot be a group. Therefore, since $S$ is an $h$-group, we have that $\mu$ is the identity congruence, and hence $\operatorname{ker} \mu=C(E)=E$ by Theorem 1 . This proves (a).

To prove (b), let $e, f, g, h \in E$ be such that $e>f$ and $g>h$. Let $\tau=\{e, f\}$ $\times\{e, f\}$. Define $\tau^{+}$on $E$ as follows: if $a, b \in E$, then $a \tau^{+} b$ if and only if $a=b$ or $a=s_{1}^{-1} u_{1} s_{1}, s_{i}^{-1} v_{i} s_{i}=s_{i+1}^{-1} u_{i+1} s_{i+1}$ for $1 \leqslant i<n, s_{n}^{-1} v_{n} s_{n}=b$, where the $s_{i}$ 's are in $S$ and $u_{i}, v_{i} \in E$ such that either $u_{i} \tau v_{i}$ or $v_{i} \tau u_{i}$ for $1 \leqslant i \leqslant n$. Note that, by [9, Lemma IV.3.2], the relation $\tau^{+}$is a normal congruence on $E$ which contains $\tau$. Then by $[9$, Theorem III.2.5] there is a congruence $\rho$ on $S$ such that the restriction of $\rho$ to $E$ is $\tau^{+}$. Since $\tau$ is not the identity congruence on $E$, then $\rho$ is not the identity congruence on $S$, and hence by hypothesis $\tau^{+}$must be the universal congruence on $E$. Hence, substituting $g, h$ for $a, b$, respectively, we have that there exist $s_{i} \in S$ and $u_{i}, v_{i} \in\{e, f\}$ such that

$$
g=s_{1}^{-1} u_{1} s_{1}, s_{i}^{-1} v_{i} s_{i}=s_{i+1}^{-1} u_{i+1} s_{i+1} \quad \text { for all } \quad 1 \leqslant i<n, s_{n}^{-1} v_{n} s_{n}=h .
$$

Without loss of generality we can assume that $u_{i} \neq v_{i}$. For $i=1, \ldots, n$, we define $p_{i}=\left(s_{i}^{-1} u_{i} s_{i}\right)\left(s_{i-1}^{-1} u_{i-1} s_{i-1}\right) \cdots\left(s_{1}^{-1} u_{1} s_{1}\right), t_{i}=s_{i} p_{i}$. Note that each $u_{i}, s_{i}^{-1} u_{i} s_{i}$, and $p_{i}$ is an idempotent. Further, as in the proof of [9, Theorem IV.3.3],

$$
t_{i}^{-1} u_{i} t_{i}=p_{i} \text { for } \quad 1 \leqslant i \leqslant n .
$$

In particular, $g=p_{1}=t_{1}^{-1} u_{1} t_{1}$, and by (1) and (2), also $t_{i}^{-1} v_{i} t_{i}=t_{i+1}^{-1} u_{i+1} t_{i+1}$ for all $1 \leqslant i<n$, and $t_{n}^{-1} v_{n} t_{n}=\left(s_{n}^{-1} v_{n} s_{n}\right) p_{n}=h p_{n} \leqslant h$. We have shown that

$$
g=t_{1}^{-1} u_{1} t_{1}, t_{i}^{-1} v_{i} t_{i}=t_{i+1}^{-1} u_{i+1} t_{i+1} \text { for all } 1 \leqslant i<n, t_{n}^{-1} v_{n} t_{n} \leqslant h .
$$

By (2) we also have

$$
t_{i}^{-1} u_{i} t_{i}=p_{i} \geqslant p_{i}\left(s_{i}^{-1} v_{i} s_{i}\right) p_{i}=t_{i}^{-1} v_{i} t_{i} \text { for all } 1 \leqslant i \leqslant n .
$$

When $\left(u_{i}, v_{i}\right)=(f, e)$ it follows from $f<e$ that $t_{i}^{-1} u_{i} t_{i} \leqslant t_{i}^{-1} v_{i} t_{i}$, which by (4) yields that $t_{i}^{-1} u_{i} t_{i}=t_{i}^{-1} v_{i} t_{i}$. Thus we may delete all terms involving $u_{i}$ and $v_{i}$ whenever $\left(u_{i}, v_{i}\right)=(f, e)$. Therefore, (b) is satisfied. 
Conversely, suppose that $S$ satisfies (a) and (b). Let $\rho$ be a congruence on $S$. If $\operatorname{tr} \rho$ is the identity congruence on $E$, then $\rho$ is idempotent separating, so $\rho \subseteq \mu$ and thus $\rho$ is the identity congruence on $S$ because $C(E)=E$. Hence, assume that $\operatorname{tr} \rho$ is not the identity congruence on $E$. Then there are distinct $e, f \in E$ such that epf. Then epef $\rho f$, so we may assume that $e>f$. Let $g, h \in E$ be such that $g>h$. By (b), we have

$$
g=t_{1}^{-1} e t_{1} \rho t_{1}^{-1} f t_{1}=t_{2}^{-1} e t_{2} \rho \cdots \rho t_{n}^{-1} f t_{n}=t
$$

so that $g \rho t$ and $t \leqslant h$. This implies that $h=h g \rho h t=t$, so that $g \rho h$. On the other hand, if $g$ and $h$ are not comparable, then $g h<g$; hence $g \rho g h$ by what has just been shown. Similarly, hogh. Therefore, again we have that $g \rho h$. Hence $\operatorname{tr} \rho$ is the universal congruence on $E$, which makes $\rho$ a group congruence.

Munn [8] has characterised congruence-free inverse semigroups as those inverse semigroups which satisfy the conditions of Theorem 2 and also have no group congruences. Hence, if we take the conditions for a congruence-free inverse semigroup $S$ and remove the condition that $S$ has no group congruences, then we obtain an $h$-group. For this reason, it is natural to think of $h$-groups as generalisations of congruence-free inverse semigroups.

\section{Special Cases}

In this section we show that the bicyclic semigroup is the only $h$-group in certain classes of semigroups.

BRUCK SEMIGROUPS. Recall that a Bruck semigroup over a monoid $T$ is defined as follows: Let $T$ be a monoid, $\alpha$ be a homomorphism of $T$ into its group of units, and $N$ be the set of all non-negative integers. On $S=N \times T \times N$ define a multiplication by

$$
(m, a, n)(p, b, q)=\left(m+n-r,\left(a \alpha^{p-r}\right)\left(b \alpha^{n-r}\right), n+q-r\right)
$$

where $r=\min \{n, p\}$ and $\alpha^{0}$ is the identity map on $T$. If $S$ is both a Bruck semigroup and an $h$-group, we say that $S$ is a Bruck $h$-group.

THEOREM 3. The bicyclic semigroup is the only Bruck h-group.

ProOF: Note that the bicyclic semigroup is the Bruck semigroup with $T$ the oneelement monoid. Conversely, suppose that $S$ is a Bruck $h$-group. Take the homomorphism $\phi:(m, a, n) \rightarrow(m, 1, n)$ where 1 is the identity of $T$. Then $\phi(S) \cong \mathcal{C}$. Since $S$ is an $h$-group, the map $\phi$ must be $1-1$, so that $S \cong \mathcal{C}$.

$w$-h-Groups. An inverse semigroup $S$ is an $\omega$-semigroup if the set of idempotents is linearly ordered with ordering the opposite of that of the non-negative integers. If $S$ is an $\omega$-semigroup and an $h$-group, we say that $S$ is an $\omega$-h-group. 
LEMma 4. [1, Theorem 1.1] If $S$ is an $h$-group, then $S$ is simple.

THEOREM 5. A semigroup $S$ is a simple $\omega$-semigroup if and only if it is a Bruck semigroup with $T$ being a chain of groups.

Proof: This follows from [10, Structure Theorem, p. 89].

THEOREM 6. The bicyclic semigroup is the only $\omega$-h-group.

Proof: Clearly, the bicyclic semigroup is an $\omega$ - $h$-group. Conversely, if $S$ is an $\omega$ $h$-group, then $S$ is simple by Lemma 4 , and hence $S$ is a Bruck semigroup by Theorem 5. The result now follows from Theorem 3 .

Recall that a Reilly semigroup is a bisimple $\omega$-semigroup. Hence, we have the following result.

COROLlary 7. The bicyclic semigroup is the only Reilly $h$-group.

Proof: By [2, Theorem 2.53] the bicyclic semigroup is bisimple, and hence is a Reilly $h$-group. Conversely, a Reilly $h$-group is an $\omega$-h-group by definition. The result now follows from Theorem 6 .

$\omega^{n}$-BISIMPLE SEMIGROUPS. Let $N$ be the set of non-negative integers and let $n$ be any positive integer. Define the reverse lexicographical order on $N^{n}$ as follows: $\left(a_{1}, a_{2}, \ldots, a_{n}\right)<\left(b_{1}, b_{2}, \ldots, b_{n}\right)$ if and only if $a_{1}>b_{1}$, or there is some index $k$, where $1<k<n$, such that $a_{1}=b_{1}, a_{2}=b_{2}, \ldots, a_{k-1}=b_{k-1}$, and $a_{k}>b_{k}$. An $\omega^{n}$-bisimple semigroup [16] is a bisimple semigroup whose idempotents are order isomorphic to the set $N^{n}$ under reverse lexicographical order.

THEOREM 8. Let $S$ be an $\omega^{n}$-bisimple semigroup, and let $k<n$. Then $S$ has a congruence $\rho$ such that $S / \rho$ is an $\omega^{k}$-bisimple semigroup.

PROOF: This is [16, Corollary 1.1].

THEOREM 9. A semigroup $S$ is an $\omega^{n}$-bisimple $h$-group if and only if $n=1$ and $S \cong \mathcal{C}$.

ProOF: By [2, Theorem 2.53] the bicyclic semigroup $\mathcal{C}$ is a bisimple $\omega^{n}$-semigroup, where $n=1$. By Theorem $6 \mathcal{C}$ is an $h$-group. Hence $\mathcal{C}$ is an $\omega^{n}$-bisimple $h$-group.

Conversely, suppose that $S$ is an $\omega^{n}$-bisimple $h$-group. By Theorem 8 we have $n=1$. Hence $S$ is an $w$-h-group. The result now follows from Theorem 6 .

In light of previous results, it is natural to ask if an $h$-group is uniquely determined by its maximal group homomorphic image. We show that this is not the case. Recall that the maximal group homomorphic image of the bicyclic semigroup is $\mathbb{Z}$, the group of integers, and that the map $\phi: \mathcal{C} \rightarrow \mathbb{Z}$ is given by $\phi\left(q^{m} p^{n}\right)=m-n$; see $[7$, Section 3.4 , Theorem 5]. 
EXAMPLE 10. There exists an h-group $S$ whose maximal homomorphic image is $\mathbb{Z}$, but $S \not \mathcal{C}$.

Let $\mathcal{C}_{1}, \mathcal{C}_{2}, \ldots, \mathcal{C}_{n}, \ldots$ be a countably infinite collection of bicyclic semigroups. We can consider each $\mathcal{C}_{j}$ to be a proper subsemigroup of $\mathcal{C}_{j+1}$ by $[2$, Theorem 2.54]. Let $S=\bigcup_{j=1}^{\infty} \mathcal{C}_{j}$, with the natural operation.

To see that $S$ is an $h$-group, let $\rho$ be a non-trivial congruence on $S$, let $a, b$ be distinct elements of $S$ such that $a \rho b$, and let $e, f$ be distinct idempotents of $S$. Find $j$ such that $a, b, e, f \in \mathcal{C}_{j}$. Since $\mathcal{C}_{j}$ is bicyclic, we get that $e \rho f$. Since $e, f$ are arbitrary idempotents, we have that $S / \rho$ is a group.

We can show that the maximal homomorphic image of $S$ is $\mathbb{Z}$ by using the same argument as that in the proof of $[7$, Section 3.4, Theorem 5]. Finally, $S \not \mathcal{C}$ because $S$ has an infinite ascending chain of idempotents.

\section{Open Problems}

Problem 1. An inverse semigroup $S$ is $E$-unitary if, when $s \in S$ and $e \in E$, then $e s \in E$ implies that $s \in E$. The congruences on $E$-unitary semigroups have been characterised; see [9, Theorem VII.2.1]. Use this characterisation to sharpen Theorem 2 for $h$-groups which are $E$ unitary.

Problem 2. Use Theorem 2 to derive the characterisations of $h$-groups in [3] or [4].

\section{REFERENCES}

[1] N. Bernstein, 'Group-like semigroups', Semigroup Forum 3 (1971/72), 58-63.

(2] A.H. Clifford and G.B. Preston, The algebraic theory of semigroups, (Vol. I) (American Mathematical Socociety, Providence, 1961).

[3] V.A. Fortunatov, 'Congruences on simple extensions of semigroups', Semigroup Forum $13(1976 / 77), 283-295$.

[4] S.M. Goberstein, 'Fundamental order relations on inverse semigroups and on their generalizations', Semigroup Forum 21 (1980), 285-328.

[5] B.A. Jensen, 'Infinite semigroups whose non-trivial homomorphs are all isomorphic', $P a$ cific J. Math 29 (1969), 583-591.

[6] G. Kowol and H. Mitsch, 'Nilpotent inverse semigroups with central idempotents', Trans. Amer. Math. Soc. 271 (1982), 437-449.

[7] M.V. Lawson, Inverse semigroups (World Scientific, New Jersey, 1998).

[8] W.D. Munn, 'Congruence-free inverse semigroups', Quart. J. Math. Oxford 2 (1974), 463-484.

[9] M. Petrich, Inverse semigroups (John Wiley and Sons, New York, 1984).

[10] M. Petrich, 'Congruences on simple w-semigroups', Glasgow Math. J. 20 (1979), 87-101.

[11] M.S. Putcha, 'Commutative semigroups whose homomorphic images in groups are groups', Semigroup Forum 3 (1971/72), 51-57. 
[12] N.R. Reilly, 'Congruence-free inverse semigroups', Proc. London Math. Soc. 3 (1976), 497-514.

[13] B. Schein, 'Pseudosimple commutative semigroups', Monatsh. Math. 91 (1981), 77-78.

[14] T. Tamura, 'Review of groups-like semigroups \#2054', by N. Bernstein, Mathematical Reviews 45 (1973), 373.

[15] T. Tamura and H. Hamilton, 'Commutative semigroups with greatest group- homomorphism', Proc. Japan Acad. 47 (1971), 671-675.

[16] R.J. Warne, 'Congruences on $\omega^{n}$-bisimple semigroups', J. Austral. Math. Soc. 9 (1969), 257-274.

[17] P. Zhu, 'On Rees congruence semigruups', Northeast. Math. J. 8 (1992), 185-191.

Department of Mathematics and Computer Science

Loyola University New Orleans

New Orleans, LA. 70118

United States of America

e-mail: tucci@loyno.edu 